\title{
Bilateral Cemented Custom-Made Total Hip Arthroplasty in Paget's Disease with Femoral Cortical Thickening and Canal Narrowing
}

\author{
Riccardo D’Ambrosi ${ }^{1}{ }^{10}$ Luca Ballini $^{1,2}$ Federico Valli ${ }^{1}$ Stefano Guarino ${ }^{1}$ Maurizio Rubino ${ }^{1}$ \\ Nicola Ursino ${ }^{1}$ \\ ${ }^{1}$ IRCCS Istituto Ortopedico Galeazzi, Milan, Italy \\ 2 Dipartimento di Scienze Biomediche per la Salute, Università degli \\ Studi di Milano, Milan, Italy \\ Address for correspondence Riccardo D'Ambrosi, MD, IRCCS Istituto \\ Ortopedico Galeazzi, Via Galeazzi 4, 20161 Milan, Italy \\ (e-mail: riccardo.dambrosi@hotmail.it).
}

Joints 2019;7:222-227.

\begin{abstract}
Keywords

- Paget's disease

- rare disease

- custom-made hip arthroplasty

- femoral cortical thickening

- canal narrowing

In this report, we present a case of a 57-year-old man complaining of pain in both his hips. Clinically, the patient reported lameness and reduction in the range of motion of the hips. Radiographic imaging showed a very narrow medullary femoral canal, confirmed by a computed tomography scan. In consideration of the narrowness of the femoral canal and cortex thickening, and due to the rarity of the clinical case, we opted to use a custom-made prosthesis. We performed total hip arthroplasty, initially on the right side. One year later, we repeated the procedure on the left side. One year after the last surgery, the patient reported well-being and continuous improvement in walking, with no complications. This case report highlights the features and the critical issues related to this kind of surgery in patients affected by Paget's disease of the bone and the importance of custom-made implants in challenging cases.
\end{abstract}

\section{Introduction}

Paget's disease of the bone (PDB) was first described in 1877 by Sir James Paget at St. Bartholomew's Hospital in London. Sir Paget described a series of middle-aged patients who presented with altered bone structures. ${ }^{1} \mathrm{He}$ named this disease "osteitis deformans" and observed that the deformities were progressively worsening and spreading to different sites. Some of these patients developed bone sarcomas, leading to their demise. ${ }^{1,2}$

The main feature of the disease is the increased bone cell activity that results in expanded bone possessing, in both sclerotic and lytic areas. The core process of PDB is the enhanced bone resorption by abnormal multinucleated osteoclasts, followed by disorganized bone formation by osteoblasts. $^{3}$ The bone tissue is metabolically active and there is a high rate of bone remodeling at the affected site, which has high blood flow. Hypervascularity is another important feature of PDB. ${ }^{4}$

PDB is the second-most frequent metabolic bone disease after osteoporosis, but despite this, it is underdiagnosed and undertreated, with many patients never having a diagnosis. ${ }^{4}$

PDB usually affects the older population; it is rare to diagnose the disease in patients under 40 years. The pathology can affect a single bone (monostotic) or many bones (polyostotic) and is often asymmetrical. ${ }^{4,5}$ It generally does not spread from bone to bone and it is rare that new bones are involved in the course of the disease. Typically, patients complain of bone pain at rest, which gets worse at night and moreover can present with gait disturbances, bony received

November 27, 2019 accepted after revision

April 19, 2021

published online

June 14, 2021
DOI https://doi.org/ 10.1055/s-0041-1730381. ISSN 2282-4324.

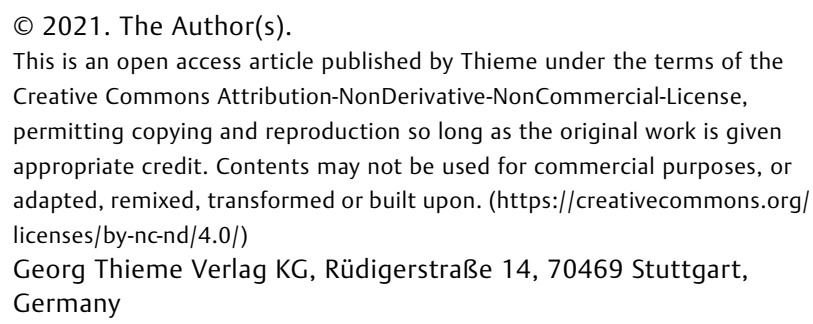


enlargements, pathological stress fractures, and secondary osteoarthritis. $^{6}$

In long bones, the osteolytic process initially develops in the proximal epiphysis. Then it progresses along the bone shaft at the rate of $8 \mathrm{~mm} /$ year. This process can be seen on Xrays as a V-shaped "lytic wedge" that progresses along the bone. In many cases, the first approach is conservative. ${ }^{7,8}$

If, despite this kind of treatment, symptoms persist, the only solution to reduce pain and functional limitation is joint arthroplasty. 9,10

One of the features of this disease is the presence of sclerotic bone. Reaming sclerotic bone can be challenging and may predispose the patient to excessive bleeding due to the hypervascular bone tissue, typical of PDB. Hypervascularity is an important issue of the disease, even more so when performing total hip arthroplasty (THA). ${ }^{11}$ THA has been consistently reported to improve the functionality of these patients. ${ }^{12}$ The optimal method for the components against the pagetoid bone has not been yet clarified. Cemented THA is reported to be a viable and accepted treatment modality for symptomatic arthritis, in patients with Paget's disease. ${ }^{13}$ Several studies reported a higher incidence of symptomatic and asymptomatic radiolucencies around the bone-cement interface of components placed against the pagetoid bone, with the possibility of increased rates of implant failure in mid- and long-term follow-up. ${ }^{12,13}$ The use of uncemented components has become the preferred method for most hip arthroplasty, reporting increased survival and a lower loosening rate, compared with cemented components. However, concerns exist regarding the implantation of cementless components against pagetoid bone, as it is not known whether the altered quality and morphology of the bone adversely influence ingrowth into uncemented implants. ${ }^{13}$

Indeed, this may constitute a problem, because bleeding can impair visualization during surgery. In postoperative days, it can cause anemization, requiring blood transfusions. It can also be a problem during cementation, compromising osteointegration and fixation of prosthesis to the affected bone. On the other hand, high bone turnover can increase the risk of early aseptic loosening in the case of uncemented implants. Further, there are intrinsic issues that characterize the pathology as the coxa vara, acetabular protrusion, or femoral bowing. ${ }^{14}$

We present a case report of a bilateral custom-made hip prostheses implant in a patient affected by Paget's disease of the hip with femoral cortical thickening and canal narrowing.

\section{Case History}

Written informed consent was obtained from the patient for publication of this case report and accompanying images.

A 57-year-old man was referred to our department because of pain in both his hips. During the medical examination, the patient complained of bilateral coxalgia for several years without a history of trauma. The pain was constant, present even at night and worse with weight bearing. Radiographic imaging suggested the presence of Paget's

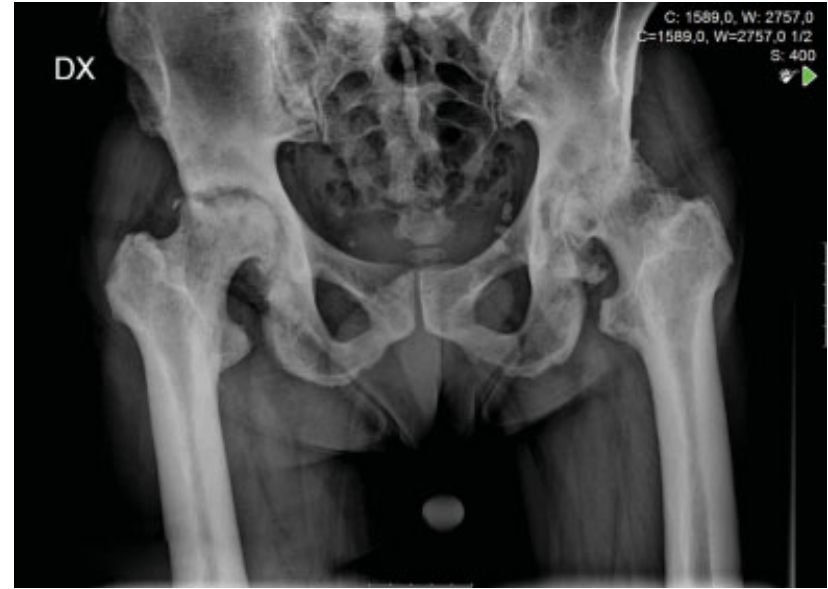

Fig. 1 Preoperative anteroposterior radiograph of the pelvis demonstrating a thickening of the corticals with narrowing of the femoral canal.

disease, confirmed also by the rise of alkaline phosphatase in blood tests (-Figs. $\mathbf{1}$ and 2A,B). Clinically, we found evident lameness and a significant reduction in range of motion (ROM), particularly in the right hip. The anteroposterior (AP) and axial radiographs showed a picture of bilateral coxarthrosis, especially on the right side, highlighting a particularly narrow femoral medullary canal with thickening of the cortex.

We therefore decided to make a computed tomography (CT) examination to study the femoral canal further and to measure it. Special scans were performed to measure and evaluate accurately the diameter of the femoral canal (-Fig. 3). CT confirmed the impossibility of using a standard femoral stem available in the market. Considering the narrowness of the femoral canal and cortex thickening, and due to the rarity of the clinical case, we opted for a custom-made prosthesis.

The choice of using a short stem prosthesis, for anatomical reasons too, was made to avoid frequent subtrochanteric fractures in patients with thickening of the femoral corticals. $^{15}$

The femoral neck preservation hip stem was accurately prepared on the basis of CT scan by Permedica S.p.A - Merate, Lecco, Italy. The stem body had a length of $55 \mathrm{~mm}$, reduced by $33 \%$ compared with a standard stem. It was also necessary to use burrs coated in physical vapor deposition (PVD) and custom-made positioning guides, to prepare the stem seat in association with the custom-made shortened rasp normally used (-Figs. 4 and 5).

\section{Surgical Technique and Postoperative Rehabilitation}

The patient was placed in lateral decubitus. The surgical access to the hip was the posterolateral one. During the surgery, we detected a reduced caliber and occlusion of the femoral canal by bone tissue. Once removed, the femoral head was sent for histological examination. We implanted a cementless Permedica Jump System Traser $56 \mathrm{~mm}$ cementless cup, stabilized with two screws and a $56 / 36 \mathrm{~mm}$ polyethylene insert with a 20-degree antidislocation shoulder 


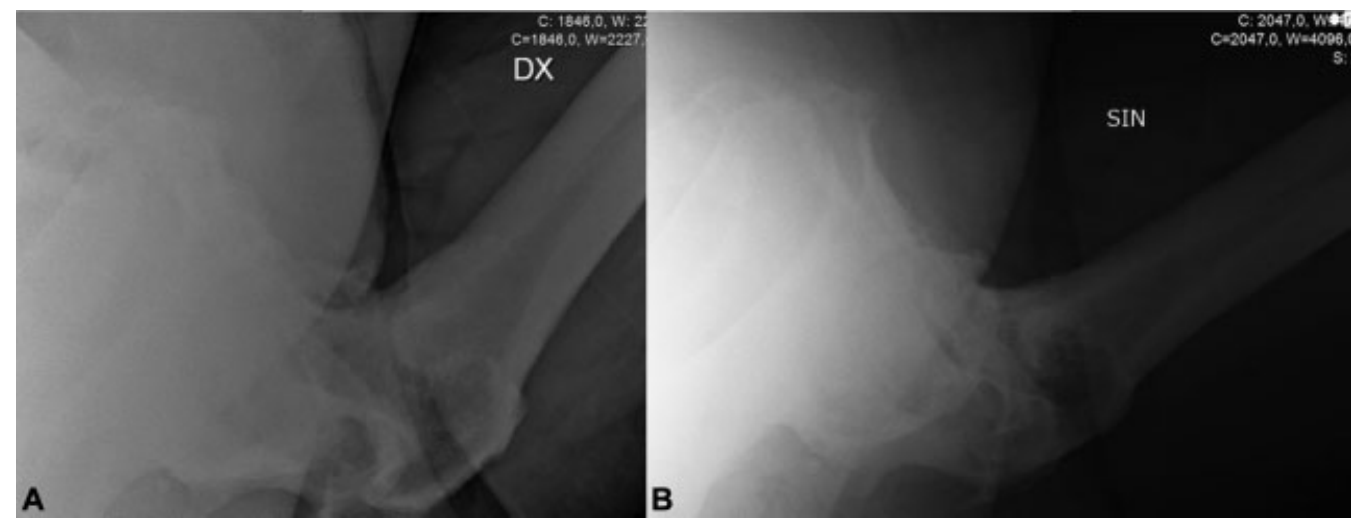

Fig. 2 Preoperative axial radiograph of the right (A) and left (B) showing alteration of the femoral canal.

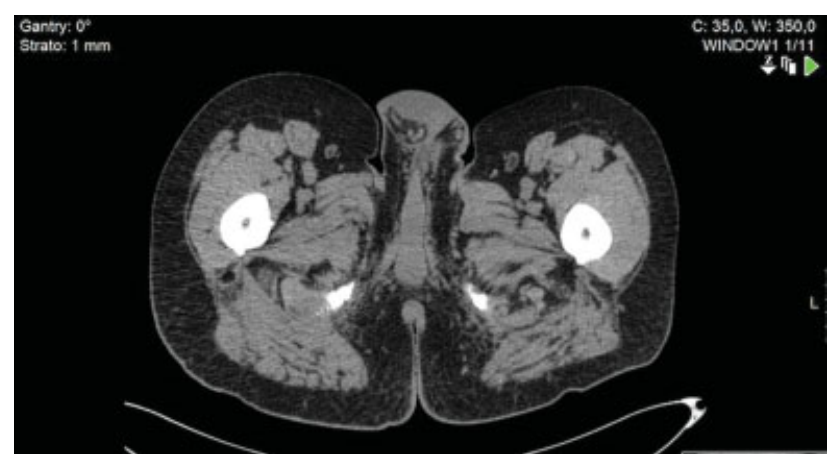

Fig. 3 Targeted computed tomography (CT) scans confirm the narrowness of the femoral canal, not suitable for normal prosthetic implants on the market.

added with vitamin $\mathrm{E}$. The femoral canal was reamed with custom-made titanium niobium nitride PVD-coated burs. There were no complications during femoral canal reaming and the custom-made femoral stem was implanted correctly, using a layer of antibiotic cement. It was necessary to use a

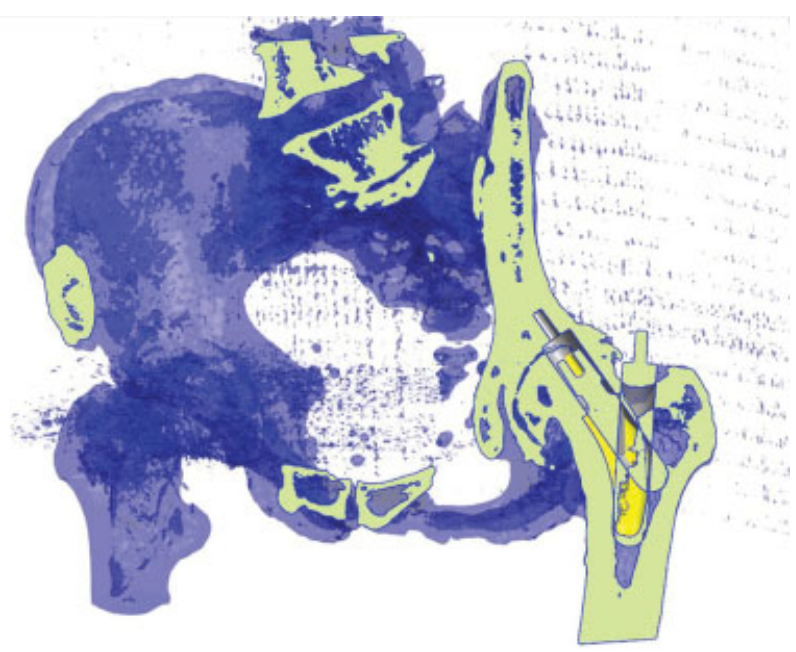

Fig. 4 Three-dimensional (3D) custom-made model obtained from computed tomography (CT) scan with the shape of the stem and the custom made $\emptyset 16 \mathrm{~mm}$ drills used for the preparation (all models have been manufactured by Permedica S.p.A.). long ceramic femoral head (36 mm diameter) (- Fig. 6A, B). The result of the histological exam confirms the diagnosis of PDB. The postoperative course was uneventful, without thermal rises and with good recovery of the ROM. Antithromboembolism prophylaxis was administered with 4,000 IU of low-molecular-weight heparin for 40 days, following our standard protocol. During the surgery, tranexamic acid was used for prophylactic purposes to limit bleeding. Hemoglobin was $13.6 \mathrm{~g} / \mathrm{dL}$ at the preoperative

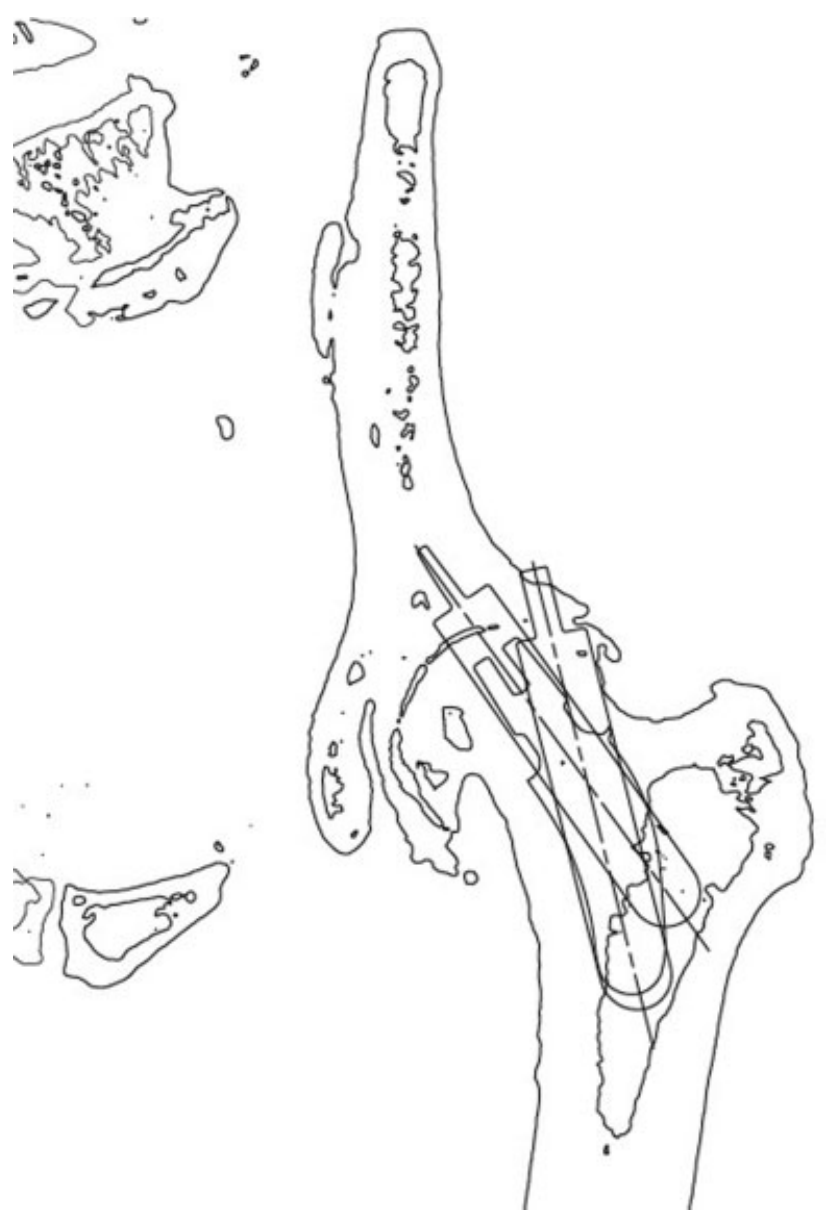

Fig. 5 Custom-made preoperative planning obtained from computed tomography (CT) scan for orientation of the burrs during the preparation of the femoral canal (all models manufactured by Permedica S.p.A.). 

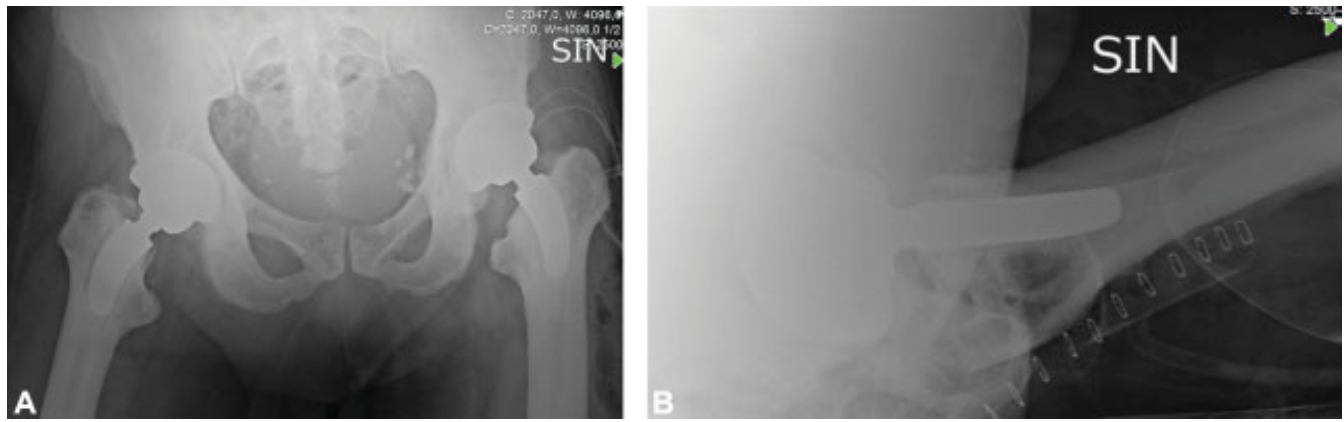

Fig. 6 (A) Anteroposterior radiograph of the pelvis showing right hip arthroplasty 1 year after surgery and left arthroplasty the day after surgery; (B) Axial view of the left hip after surgery.

blood tests. On the first postoperative day, the hemoglobin value was 10.2 and the lowest value recorded during hospitalization was $7.5 \mathrm{~g} / \mathrm{dL}$ on the sixth postoperative day, which necessitated a blood transfusion. Details of blood tests after the two surgeries are reported in -Table 1.

On the first day, the patient started regular physiotherapy, consisting of mobilization in bed, muscle strengthening, and verticalization. On the third postoperative day, the patient was transferred to the rehabilitation department. Upon admission to the rehabilitation unit, the Barthel index was 63 and the Functional Independence Measure (FIM) score was 95 . On the 13th postoperative day, he was discharged with $9.4 \mathrm{~g} / \mathrm{dL}$ of hemoglobin, a Barthel score of 98 , and an FIM score of 122 .

One year after surgery, the patient was hospitalized for left hip arthroplasty. A new CT scan confirmed the reduced caliber of the femoral canal. A second custom-made prosthesis was then prepared by the same company (Permedica S.p.A), maintaining the same characteristics of the right one. The surgical technique and the final implants were the same as in the surgery performed a year before (-Fig. 6A, B). The only difference concerned the use of screws to fix the acetabular cup, which was not necessary for the left hip.

In both hips, we opted for a long ceramic head, unlike the preoperative plan. This was an intraoperative choice due to the partial subversion of the normal anatomy, to the alteration of the periarticular soft tissues; the choice of a longer head guarantees greater stability and a restoration of normal anatomy, as far as possible.

Also, in this case, tranexamic acid was used during the operation. In preoperative blood tests, hemoglobin was $14.3 \mathrm{~g} / \mathrm{dL}$, while on the first postoperative day it was $13.4 \mathrm{~g} / \mathrm{dL}$. The lowest hemoglobin level was reached on the sixth postoperative day, with a value of $7.5 \mathrm{~g} / \mathrm{dL}$, which did not require transfusion. As during the previous hospitalization, the patient started physiotherapy on the first day, and on the third day, was transferred to the rehabilitation department. As for the previous hospitalization and in consideration of the results of the blood tests, no specific drug therapy for Paget's disease has been indicated to the patient.

Upon admission to the rehabilitation unit, the Barthel index was 83 and the FIM index was 108. On the 14th postoperative day, he was discharged with a hemoglobin value of $8.8 \mathrm{~g} / \mathrm{dL}$, a score of Barthel of 100, and a FIM value of 123.

Respectively 30 and 18 months after the first and second surgery, the patient reported a Harris Hip Score of 90, PCS-12 (physical score) of 43.44, and MCS-12 (mental score) of 52.67. No episodes of dislocation, femoral fractures, or infection were reported throughout the follow-up.

Table 1 Pre- and postoperative blood tests

\begin{tabular}{|l|l|l|l|l|l|l|}
\hline & \multicolumn{2}{|l|}{ Right hip } & Left hip \\
\cline { 2 - 7 } & $\begin{array}{l}\text { Day before } \\
\text { surgery }\end{array}$ & $\begin{array}{l}\text { First day after } \\
\text { surgery }\end{array}$ & $\begin{array}{l}\text { Second day } \\
\text { after surgery }\end{array}$ & $\begin{array}{l}\text { Day before } \\
\text { surgery }\end{array}$ & $\begin{array}{l}\text { First day } \\
\text { after surgery }\end{array}$ & $\begin{array}{l}\text { Second day } \\
\text { after surgery }\end{array}$ \\
\hline Hemoglobin, g/dL & 13.6 & 10.2 & 9.2 & 14.3 & 13.4 & 9.8 \\
\hline White blood cells, $10^{3} / \mathrm{\mu L}$ & 7.46 & 10.78 & 9.18 & 6.30 & 11.79 & 8.66 \\
\hline Calcium, mg/dL & 8.9 & 7.8 & 7.8 & 9.3 & 8.6 & 8.2 \\
\hline Magnesium, mmol/L & 0.75 & 0.72 & 0.79 & 0.77 & 0.88 & 0.82 \\
\hline Phosphates, mmol/L & 0.95 & 1.04 & 0.87 & 0.96 & 0.91 & 0.88 \\
\hline Potassium, mEq/L & 3.93 & 3.99 & 3.80 & 4.25 & 3.87 & 3.71 \\
\hline C-reactive protein, $\mathrm{mg} / \mathrm{dL}$ & 0.76 & 4.24 & 4.50 & 0.29 & 6.30 & 6.80 \\
\hline
\end{tabular}




\section{Discussion}

To our knowledge, there are no other published reports documenting the bilateral implant of cemented custommade hip arthroplasty in patients with Paget's disease with canal narrowing and cortical thickening.

In this situation, the possibility of using a custom-made implant allowed the intervention to take place, as otherwise, the prosthetic models available on the market could not be used.

The use of custom-made prostheses, despite the high costs, is increasingly adopted, particularly in the case of rare diseases that alter the quality and shape of the bone. A recent review has analyzed the use of custom-made prostheses in patients suffering from a congenital disease of the hip. ${ }^{16}$ The article highlights, as in our case, the importance of preoperative planning when a custom-made arthroplasty is performed: initial planning involves a complete pelvis X-ray examination with the central beam toward the symphysis pubis, and an axial X-ray of the hip. A CT evaluation of the hip and of the femur must always be performed. ${ }^{16}$ The femoral stem is manufactured using a computer numerical control machine. It is important to restore the normal biomechanics of the hip joint, correcting the defects of proximal femoral anatomy. Custom- made implants aim to restore the normal anteversion of 15 degrees. The choice of geometrical forms and materials of the components is also crucial. The choice of materials affects biocompatibility and osteointegration capacity.

The concept of femoral custom-made stem is based on the verification that it is better to fit the prosthesis to the femur, rather than shaping the femur to fit the prosthesis. ${ }^{17}$ Individual stem designs have a more favorable strain distribution, compared with standard anatomical stems. ${ }^{18}$ Existing evidence from several clinical and experimental studies outlines the importance of geometrical fitting of the uncemented femoral stems in patients with THA. ${ }^{18-21}$ Inadequate canal filling has been considered the main cause of stem failure. ${ }^{22}$ Lack of bonding between the bone and the implant will lead to progressive stress shielding, bone remodeling, development of a fibrous membrane at the interfaces, and finally an unstable prosthesis.

The specific problems of the surgical technique concern the implantation of both the acetabular component and the femoral component. As regards the acetabular component, the problems consist mainly of the presence of sclerotic bone and protrusio. To avoid the problem of sclerosis, some authors suggest using high speed burr. To obtain the optimal center of rotation of the hip, a lateral acetabular liner can be used, while a cage can be useful to avoid acetabular prortrusion. ${ }^{7,8}$

As far as the femoral component is concerned, the risks are related more to the malposition of the stem. Preoperative planning is crucial at this stage in determining the height of the osteotomy.

Another issue is cementation. There are authors who report good results with cementless implants and others who report equally good results with cemented ones. ${ }^{14}$

Some reports show that the failure rate with revision of the prosthesis in case of use of cement reaches 15\% and that the failure rate is related to plasma alkaline phosphatase levels, a measure of disease activity. ${ }^{23-25}$

A recent review analyzed the clinical and functional outcomes following THA in patients with Paget's disease. ${ }^{14}$ Eight studies involving 358 hips were reviewed. The mean age was 70.4 years and follow-up was 8.3 years. There were 247 cemented THAs (69\%), 105 uncemented THAs (29\%), and 6 hybrid THAs (2\%). All studies reported significant improvement in hip function following THA. There were 19 cases of aseptic loosening (5\%) at a mean of 8.6 years. Three cases occurred in the uncemented cohort (3\%) at a mean of 15.3 years and 16 cases developed in the cemented group (6\%) at a mean of 7.5 years. There were 27 revisions in the 358 cases (8\%) occurring at a mean of 7 years. Six revisions occurred in the uncemented cohort (6\%) at a mean of 8.6 years and 21 in the cemented cohort (9\%) at a mean of 6.5 years. $^{14}$

In literature, there is still no gold standard on the use of cemented or noncemented prostheses in patients with Paget's disease.

A systematic review published in 2017 showed similar functional outcomes and survivorship using either cementless or cemented components, with a similar rate of overall revisions. However, cementless components resulted in a decreased incidence of aseptic loosening and revision due to aseptic loosening. ${ }^{13}$

There was a recent report of PDB in an 84-year-old woman, which was initially identified as avascular necrosis of the hip, for which she underwent THA. During follow-up, the patient complained about hip pain, and in a few months, she was not able to walk because of early loosening with bone destruction. Radiological and laboratory exams were performed with normal results, except for AP. After treatment with bisphosphonates, the hip pain was relieved, but the patient refused other surgery to the hip. ${ }^{26}$

Finally, in consideration of the patient's blood tests, we decided not to administer bisphosphonate therapies in the postoperative period. In this way, we also tried to reduce the risk of bisphosphonate-related atypical femoral fracture; in fact, most studies show that the use of bisphosphonate increases the incidence of atypical femoral fracture, and the incidence increases with duration of use, especially after 3 years. $^{27}$

THA in patients with Paget's disease can be a technically difficult procedure with many complications and should be performed only by experienced hip surgeons. In the case of narrowing of the canal and cortical thickening, the custommade prosthesis has proven to be a safe and effective alternative, allowing surgical intervention and clinical improvements even in the most complex cases.

Conflict of Interest

None declared. 


\section{References}

1 Coppes-Zantinga AR, Coppes MJ. Sir James Paget (1814-1889): a great academic Victorian. J Am Coll Surg 2000;191(01):70-74

2 Paget J. On a form of chronic inflammation of bones (osteitis deformans). Med Chir Trans 1877;60:37-64, 9

3 van Staa TP, Selby P, Leufkens HG, Lyles K, Sprafka JM, Cooper C. Incidence and natural history of Paget's disease of bone in England and Wales. J Bone Miner Res 2002;17(03):465-471

4 Ralston SH, Corral-Gudino L, Cooper C, et al. Clinical guidelines on Paget's disease of bone. J Bone Miner Res 2019;34(12):2327-2329

5 Al Nofal AA, Altayar O, BenKhadra K, et al. Bone turnover markers in Paget's disease of the bone: a systematic review and metaanalysis. Osteoporos Int 2015;26(07):1875-1891

6 Roodman GD, Windle JJ. Paget disease of bone. J Clin Invest 2005; 115(02):200-208

7 Cundy T. Paget's disease of bone. Metabolism 2018;80:5-14

8 Al-Rashid M, Ramkumar DB, Raskin K, Schwab J, Hornicek FJ, Lozano-Calderón SA. Paget disease of bone. Orthop Clin North Am 2015;46(04):577-585

9 Lee GC, Sanchez-Sotelo J, Berry DJ. Total knee arthroplasty in patients with Paget's disease of bone at the knee. J Arthroplasty 2005;20(06):689-693

10 Tyagi V, Lajam C, Deshmukh AJ. Total hip arthroplasty in Paget's disease a review. Bull Hosp Jt Dis (2013) 2016;74(04):270-274

11 Merlotti D, Gennari L, Martini G, Nuti R. Current options for the treatment of Paget's disease of the bone. Open Access Rheumatol 2009;1:107-120

12 Makaram N, Woods L, Beattie N, Roberts SB, Macpherson GJ. Longterm outcomes following total hip and total knee arthroplasty in patients with Paget's disease of bone (PDB) - a national study. Surgeon 2020;18(06):335-343

13 Hurley TE, Cashman J, Synnott KA, O’Byrne JM, Lyons FG. Cemented versus cementless total hip arthroplasty in Paget's disease of bone: a systematic review. J Hip Surg 2017;1:87-92

14 Hanna SA, Dawson-Bowling S, Millington S, Bhumbra R, Achan P. Total hip arthroplasty in patients with Paget's disease of bone: a systematic review. World J Orthop 2017;8(04):357-363

15 Moya-Angeler J, Zambrana L, Westrich GH, Lane JM. Atypical femoral fracture post total hip replacement in a patient with hip osteoarthritis and an ipsilateral cortical thickening. Hip Int 2016;26(02):e19-e23
16 Tsiampas DT, Pakos EE, Georgiadis GC, Xenakis TA. Custom-made femoral implants in total hip arthroplasty due to congenital disease of the hip: a review. Hip Int 2016;26(03):209-214

17 Bargar WL. Shape the implant to the patient. A rationale for the use of custom-fit cementless total hip implants. Clin Orthop Relat Res 1989;(249):73-78

18 Aamodt A, Lund-Larsen J, Eine J, Andersen E, Benum P, Husby OS. Changes in proximal femoral strain after insertion of uncemented standard and customised femoral stems. An experimental study in human femora. J Bone Joint Surg Br 2001;83(06): 921-929

19 Helm CS, Greenwald AS. The rationale and performance of modularity in total hip arthroplasty. Orthopedics 2005;28(9, Suppl):s1113-s1115

20 Jasty M, O'Connor DO, Henshaw RM, Harrigan TP, Harris WH. Fit of the uncemented femoral component and the use of cement influence the strain transfer the femoral cortex. J Orthop Res 1994;12(05):648-656

21 Sedlacek RC, O'Connor DO, Lozynsky AJ, Harris WH. Assessment of the symmetry of bone strains in the proximal femoral medial cortex under load in bilateral pairs of cadaver femurs. J Arthroplasty 1997;12(06):689-694

22 Kobayashi S, Terayama K. Factors influencing survivorship of the femoral component after primary low-friction hip arthroplasty. J Arthroplasty 1992;7(Suppl):327-338

23 Menéndez-Bueyes LR, Soler Fernández MD. Paget's disease of bone: approach to its historical origins. Reumatol Clin 2017;13 (02):66-72

24 Muschitz C, Feichtinger X, Haschka J, Kocijan R. Diagnosis and treatment of Paget's disease of bone : a clinical practice guideline. Wien Med Wochenschr 2017;167(1-2):18-24

25 Lusty PJ, Walter WL, Walter WK, Zicat B. Cementless hip arthroplasty in Paget's disease at medium-term follow-up (average of 6.7 years). J Arthroplasty 2007;22(05):692-696

26 Crego-Vita D, Aedo-Martín D, Sánchez-Pérez C. Case report of early aseptic loosening of total hip arthroplasty in monostotic Paget disease, a diagnostic challenge. Int J Surg Case Rep 2016; 24:215-218

27 Silverman S, Kupperman E, Bukata S. Bisphosphonate-related atypical femoral fracture: managing a rare but serious complication. Cleve Clin J Med 2018;85(11):885-893 